\title{
USE OF THE LAPLACE TRANSFORM TECHNIQUE FOR SIMPLE KINETIC PARAMETERS EVALUATION. APPLICATION TO THE ADSORPTION OF A PROTEIN ON POROUS BEADS
}

\author{
J. MEMBREZ,* P. P. INFELTA ${ }^{\dagger}$ and A. RENKEN*‡ \\ *Institute of Chemical Engineering, ${ }^{\dagger}$ Institute of Physical Chemistry, Swiss Federal Institute of \\ Technology, CH-1015 Lausanne, Switzerland
}

(Received 27 March 1995; accepted 26 February 1996)

\begin{abstract}
The kinetics of the adsorption of human serum albumin (HSA) onto spherical resin beads (Blue Sepharose CL-6B) in a closed stirred tank have been investigated. The differential equation with appropriate boundary conditions at the grain outer surface may be solved numerically or to various degrees of approximation. Using the Laplace transform technique to solve the equation of interest, we are able to obtain the exact solution to the problem, in the Laplace domain. We assume that equilibrium is described by a linear adsorption isotherm and that the adsorption rate is very rapid compared to diffusion in the adsorbent particles. A functional description of the experimental data in the time domain allows us to compute the corresponding Laplace transform and fit it to the exact solution, to obtain the film mass transfer coefficient, $k_{f}$, and the effective diffusion coefficient, $D_{e}$. The main advantage of this Laplace transform technique is that time-consuming numerical approaches are not needed. The two parameters are rapidly and easily found via two algebraic fits, one in the time domain followed by another in the Laplace domain. Using the parameters thus obtained, a numerical solution of the problem is in good agreement with the experimental data. Copyright (C) 1996 Elsevier Science Ltd
\end{abstract}

Keywords: Protein, adsorption, kinetics, parameters, estimation, Laplace transform.

\section{INTRODUCTION}

The downstream processing of biochemical compounds as well as the industrial effluents treatment for pollution control use adsorbents onto which the target molecules are adsorbed. These molecules are then recovered or discarded by means of a desorbing agent. For the proper design of an industrial process, it is important to be able to predict the extent of adsorption on the substrates used for that purpose. In order to achieve this goal, we must find a kinetic model describing the phenomenon. This requires the knowledge of transport and equilibrium parameters that are closely related to external mass transport towards the adsorbing particles, intraparticle diffusion of the target molecules through the pores of the adsorbent, and the adsorption process of the molecules at the adsorption sites at the surface of the pore walls.

This is a rather difficult problem that is often solved numerically (Arve and Liapis, 1987; Casillas et al., 1993; Firouztale et al., 1992; Horstmann and Chase, 1989). In the case of the adsorption in a closed stirred tank only very simple situations give rise to analytical solutions that are easy to use (Chase, 1984; McKay et al., 1983; Somers et al., 1989).

Ruthven (1984) reviews in great detail the mathematical solutions available for a wide range of

${ }^{\ddagger}$ Author to whom correspondence should be addressed. adsorption kinetic models. Analytical solutions are provided for both batch and column systems. In the case of the batch systems, the solutions presented are insufficient since only diffusion is accounted for. Analytical solutions are provided for many of the situations commonly encountered for the adsorption kinetics in columns, including the famous Rosen's solution (Rosen, 1952) that accounts for both intraparticle diffusion and film mass transfer resistance with the assumption of a plug flow and a linear adsorption isotherm. All the solutions provided have been obtained by Laplace transformation. They have the great disadvantage of being cumbersome expressions that are not easy to compute to lead to a rapid estimation of the kinetic parameters.

Another widely used approach is the method of moments presented in several publications (Arnold $e t$ al., 1985; Boyer and Hsu, 1992b; Ruthven, 1984) and has been applied to numerous chromatographic systems. The method is based on the analysis of the response to a tracer pulse at the inlet of a column packed with the adsorbent of interest. The adsorption isotherm is supposed to be linear. Moreover, the assumptions of the model include axial dispersion of the liquid, film mass transfer resistance as well as diffusion inside the pores of the adsorbent. The first and second moments of the response can be theoretically calculated by inversion of the corresponding expressions in the Laplace domain. The kinetic parameters are 
obtained by linear regression (i.e. plot of the second moment of the distribution divided by the inverse of the interstitial velocity against the inverse of the interstitial velocity). The slope is proportional to the axial dispersion coefficient and the intercept is a combination of the effective diffusion coefficient and the film mass transfer coefficient. In order to calculate the effective diffusion coefficient, the film mass transfer coefficient must be estimated by an adequate correlation. In this method, the kinetic parameters are not fitted individually. They must be calculated from the intercept of a linear regression which may be imprecise when only few experimental data points are available, especially near the intercept. These measurements may be difficult to obtain because of interstitial velocity limitation. Another disadvantage has been reported by Christoffel (1989): the axial dispersion coefficient as well as the tracer input time depend on the reactor configuration. If the reactor model is not correct, the kinetic parameters that do not depend on the reactor configuration are affected and cannot be transferred to another configuration. To avoid this problem, we chose to perform our study in a well characterized closed system. The independently obtained kinetic parameters can then be used for the design of separation columns.

An elegant method for the determination of the kinetic parameters is the inversion of the solution found in the Laplace domain by a fast Fourier transform method. Boyer and Hsu (1992b) apply it successfully to check the validity of the parameters found by the method of moments. This inversion can be coupled to an optimization algorithm in order to obtain the kinetic parameters from a set of data in the time domain.

Our purpose is to propose here another solution to this problem that is complete, easy to handle and that does not require the numerical resolution of the problem, either to solve the set of partial differential equations or to invert the solution found in the Laplace domain. We use the Laplace transform technique to solve the set of partial differential equations of interest and thus obtain, under some basic assumptions, the exact solution in the Laplace domain. Because of the analytical complexity, the actual solution in the time domain is not readily available. However, using a functional description of the time-dependent measurements, we are able to obtain the Laplace transform of the data. It is then possible to fit the latter to the exact solution of the problem and thus obtain the kinetic parameters of interest. In the present paper, the application of this method to the adsorption of human serum albumin (HSA) on Blue Sepharose CL-6B in a closed stirred tank is described. The system provides a relatively inexpensive specific adsorption system that is close to the affinity techniques that are widely used. In order to test the validity of the technique, the kinetic parameters obtained by the Laplace transform technique are then used for a numerical simulation of the integral adsorption process.

\section{THEORETICAL BASIS AND MODEL}

\subsection{Generalities}

We assume that the spherical particles, once swollen with the solvent, all have the same geometrical radius, $R$. The porosity, $\varepsilon$, is the ratio of the volume of liquid contained in a particle accessible to the protein, $V_{\text {void }}$, to its total volume, $V_{p}$.

$$
\varepsilon=\frac{V_{\text {void }}}{V_{p}}
$$

At the beginning of the experiment, the particles are totally free of protein. The amount of protein contained in one particle varies with time because of protein transfer through the surrounding liquid film, diffusion in the liquid contained in the pores, as well as adsorption on the walls of the pores. The following quantities are relevant to the model. $c_{i}(r, t)$ : amount of protein in the liquid at distance $r$ from the center of a particle, at time $t$, inside, averaged over all directions, per unit volume of swollen particle. $c_{l i}(r, t)$ : amount of protein in the liquid contained in the pores of a particle at distance $r$ from its center and at time $t$, averaged over all directions, per unit volume of liquid in a swollen particle. The relation between $c_{i}(r, t)$ and $c_{l i}(r, t)$ is

$$
c_{i}(r, t)=\varepsilon c_{l i}(r, t)
$$

The mass balance written for an elementary volume of swollen particle, at distance $r$ from the center of a particle and at time $t$, leads to

$$
\rho_{p} \frac{\partial q_{i}}{\partial t}+\varepsilon \frac{\partial c_{l i}}{\partial t}=\varepsilon D_{l}\left(\frac{\partial^{2} c_{l i}}{\partial r^{2}}+\frac{2}{r} \frac{\partial c_{l i}}{\partial r}\right)
$$

where $q_{i}(r, t)$ is the amount of adsorbed protein on the pore walls at distance $r$ from the center of a particle, at time $t$, averaged over all directions, per unit mass of dry resin, $\rho_{p}$ is the mass of dry resin per unit volume of swollen resin and $D_{l}$ is the diffusion coefficient of the protein diffusing in the liquid of the pores.

As adsorption is generally a fast process, we assume that the reaction at the surface of the pores is instantaneous and that the amount of protein adsorbed, $q_{i}(r, t)$, is proportional to the protein concentration in the liquid of the pores. Thus

$$
q_{i}(r, t)=K c_{l i}(r, t)
$$

The differential eq. (3) then becomes

$$
\rho_{p} K \frac{\partial c_{l i}}{\partial t}+\varepsilon \frac{\partial c_{l i}}{\partial t}=\varepsilon D_{l}\left(\frac{\partial^{2} c_{l i}}{\partial r^{2}}+\frac{2}{r} \frac{\partial c_{l i}}{\partial r}\right)
$$

and finally in terms of $c_{i}$

$$
\left(1+\frac{\rho_{p} K}{\varepsilon}\right) \frac{\partial c_{i}}{\partial t}=D_{l}\left(\frac{\partial^{2} c_{i}}{\partial r^{2}}+\frac{2}{r} \frac{\partial c_{i}}{\partial r}\right) .
$$


The amount of protein per unit volume of swollen resin obeys a diffusion equation

$$
\begin{aligned}
& \frac{\partial c_{i}}{\partial t}=D\left(\frac{\partial^{2} c_{i}}{\partial r^{2}}+\frac{2}{r} \frac{\partial c_{i}}{\partial r}\right) \\
& \text { where } D=\frac{D_{l}}{1+\rho_{p} K / \varepsilon} .
\end{aligned}
$$

\subsection{Time-dependent problem}

Let us find an expression for $c_{\text {bulk }}(t)$, the bulk concentration of the protein, which is the measurable quantity in our experiment. For this, we need to find $c_{i}(r, t)$, the solution to eq. (7) with boundary conditions relevant to our problem, describing the averaged protein concentration inside a particle. Making use of eqs (6) and (7), let us express $\phi(t)$, the amount of protein entering one particle per unit time

$$
\begin{aligned}
\Phi(t) & =\iiint_{V_{p}}\left(\rho_{p} \frac{\partial q_{i}}{\partial t}+\varepsilon \frac{\partial c_{l i}}{\partial t}\right) \mathrm{d} V \\
& =\iiint_{V_{p}} D_{l}\left(\frac{\partial^{2} c_{i}}{\partial r^{2}}+\frac{2}{r} \frac{\partial c_{i}}{\partial r}\right) \mathrm{d} V .
\end{aligned}
$$

This can be further simplified by making use of Green's theorem:

$$
\Phi(t)=D_{l} \iiint_{V_{p}} \operatorname{div} \overrightarrow{\operatorname{grad}} c_{i} \mathrm{~d} V=D_{l} \iiint_{S_{p}} \overrightarrow{\operatorname{grad}} c_{i} \mathrm{~d} \vec{S}
$$

Taking into account the spherical symmetry of the problem, we obtain

$$
\Phi(t)=4 \pi R^{2} D_{l}\left[\frac{\partial c_{i}(r, t)}{\partial r}\right]_{r=R} .
$$

The flux at the boundary of a particle, per geometrical unit area of particle, is controlled by mass transfer and assumed to be proportional to the difference in protein concentration in the bulk of the solution and in the pores at the edge of the particles. At the onset of the adsorption, no protein is present in the particles. This leads to

$$
\begin{aligned}
& D_{l}\left[\frac{\partial c_{l i}(r, t)}{\partial r}\right]_{r=R}=k_{l}\left[c_{\text {bulk }}(t)-c_{l i}(R, t)\right] \\
& \text { or } \\
& D_{e}\left[\frac{\partial c_{i}(r, t)}{\partial r}\right]_{r=R}=k_{f}\left[\varepsilon c_{\text {bulk }}(t)-c_{i}(R, t)\right] \\
& c_{i}(r, 0)=0 \\
& \quad \text { with } k_{f}=k_{l} \cdot \varepsilon \\
& \text { and } D_{e}=D_{l} \cdot \varepsilon
\end{aligned}
$$

where $c_{\text {bulk }}(t)$ is the amount of protein per unit volume of free solvent (bulk concentration), $k_{f}$ is the liquid film mass transfer coefficient, $k_{l}$ is the modified liquid film mass transfer coefficient, and $D_{e}$ is the effective diffusion coefficient.

At equilibrium, there is no more driving force for diffusion since the liquid concentration of the solute is the same throughout the system.

The amount of protein present at time $t$ inside one particle, $n_{\text {part }}(t)$, whether adsorbed or in the liquid of the pores, is given by

$$
n_{\text {part }}(t)=\int_{0}^{t} \Phi(t) \mathrm{d} t
$$

The total amount of protein inside all the particles, $n_{i}(t)$, at time $t$, is simply given by

$$
n_{i}(t)=N_{\text {part }} n_{\text {part }}(t)=N_{\text {part }} \int_{0}^{t} \Phi(t) \mathrm{d} t
$$

where $N_{\text {part }}$ is the total number of particles in the sample.

Now taking into account the fact that the total amount of protein, $n$, remains constant during the experiment, we have

$$
\begin{aligned}
n & =n_{\text {bulk }}(t)+n_{i}(t) \Rightarrow c_{\text {bulk }}(t) \\
& =\frac{n}{V_{\text {bulk }}}-\frac{N_{\text {part }}}{V_{\text {bulk }}} \int_{0} \Phi(t) \mathrm{d} t
\end{aligned}
$$

where $n_{\text {bulk }}(t)$ is the amount of protein in the bulk of the solution. Another expression for $c_{\text {bulk }}(t)$ is obtained from the boundary condition (11). We have

$$
\begin{gathered}
4 \pi R^{2} D_{l}\left[\frac{\partial c_{i}(R, t)}{\partial r}\right]_{r=R}=4 \pi R^{2} k_{l}\left[\varepsilon c_{\text {bulk }}(t)-c_{i}(R, t)\right] \\
=\Phi(t) c_{\text {bulk }}(t)=\frac{n_{\text {bulk }}(t)}{V_{\text {bulk }}}=\frac{c_{i}(R, t)}{\varepsilon}+\frac{\Phi(t)}{4 \pi R^{2} k_{l} \varepsilon}
\end{gathered}
$$

Both expressions for $c_{\text {bulk }}(t)$ [eqs (14)-(15)] must be equivalent and this, as we shall see, unequivocally determines $c_{i}(R, t)$. We can now proceed towards the solution of the problem.

\subsection{Solution in the Laplace domain}

We use the Laplace transform technique to solve the partial differential eq. (7). Let $\bar{c}$ be the Laplace transform of $c_{i}(r, t)$ and let us take the Laplace transform of eq. (7); we have

$$
\begin{aligned}
\bar{c}=\int_{0}^{\infty} \mathrm{e}^{-s t} c_{i}(r, t) \mathrm{d} t & \Rightarrow D\left(\frac{\mathrm{d}^{2} \bar{c}}{\mathrm{~d} r^{2}}+\frac{2}{\mathrm{r}} \frac{\mathrm{d} \bar{c}}{\mathrm{~d} r}\right) \\
& -s \bar{c}+c_{i}(r, 0)=0 .
\end{aligned}
$$

The amount of protein per unit volume of swollen particle at $r=R$ is $c_{i}(R, t)$. Then, the solution of eq. (16) is given by

$$
\bar{c}=\bar{c}(R) \frac{R}{r} \frac{\mathrm{e}^{r \sqrt{s / D}}-e^{-r \sqrt{s / D}}}{e^{R \sqrt{s / D}}-e^{-R \sqrt{s / D}}}
$$


where $\bar{c}(R)$ is the Laplace transform of $\mathcal{c}_{i}(R, t)$. The exact expression for $c_{i}(R, t)$ must be selected so that the boundary conditions (11) are verified.

Let us take the Laplace transform of $c_{\text {bulk }}(t)$ as expressed by eqs (14) and (15). We obtain

$$
\bar{c}_{\text {bulk }}=\frac{1}{V_{\text {bulk }}}\left(\frac{n}{s}-N_{\text {part }} \frac{\bar{\Phi}}{s}\right)=\frac{\bar{c}(R)}{\varepsilon}+\frac{\bar{\Phi}}{4 \pi R^{2} k_{l} \varepsilon}
$$

where $\bar{\Phi}$ is the Laplace transform of $\Phi(t)$. The Laplace transform of $\Phi(t)$ can be obtained from eq. (17) and from the Laplace transform of eq. (10) which is

$$
\bar{\Phi}=4 \pi R^{2} D_{l}\left(\frac{\mathrm{d} \bar{c}}{\mathrm{~d} r}\right)_{r=R} .
$$

Equation (17) allows the calculation of the spatial derivative of $\bar{c}$ at $r=R$, giving an expression for $\bar{\Phi}$ as a function of $\bar{c}(R)$. Inserting this expression into eq. (18) leads through somewhat tedious calculation to the expression of $\bar{c}(R)$ which can then be used in eq. (17). This provides the final expression for $\bar{c}_{\text {bulk }}$ as a function of the kinetic parameters of the model and $s$
The parameters $a, b, f$ and $d$ are obtained by fitting the experimental data to eq. (21) (least-squares fitting). Note that the parameter $a$ theoretically equals the bulk concentration at equilibrium, $c_{\text {bulk }}$ (eq). Equation (22) provides numerical values for the Laplace transform of $c_{\text {bulk }}(t)$. We recall the boundaries theorems:

$$
\begin{aligned}
& \lim _{s \rightarrow \infty} s \bar{c}_{\text {bulk }}=\lim _{t \rightarrow 0} \bar{c}_{\text {bulk }}(t) \\
& \lim _{s \rightarrow 0} s \bar{c}_{\text {bulk }}=\lim _{t \rightarrow \infty} \bar{c}_{\text {bulk }}(t)
\end{aligned}
$$

which give the limiting values of $c_{\text {bulk }}(t)$ at short and long times. The values of $\bar{c}_{\text {bulk }}$ span many orders of magnitude and it is essential to perform the fitting procedure on weighted values of $\bar{c}_{\text {bulk }}$ for a distribution of $s$ values which has to be appropriate. The above limiting theorems indicate that $s \bar{c}_{\text {bulk }}$ takes values corresponding to the experimentally measured concentrations. We fit the values of $s c_{\text {bulk }}$ obtained from eq. (22) to expression (20), for values of $s$ selected to be evenly distributed on a logarithmic scale. The

$\bar{c}_{\text {bulk }}=\frac{n}{V_{\text {bulk }}}\left\{\frac{\left[\mathrm{e}^{-2 A}(1-B i+A)\right]-1+B i+A}{\left[\mathrm{e}^{-2 A}\left(\varepsilon k_{l} a_{l}+\varepsilon k_{l} a_{l} A+s-s B i+s A\right)\right]-\varepsilon k_{l} a_{l}+\varepsilon k_{l} a_{l} A-s+s B i+s A}\right\}$

where

$A=R \sqrt{\frac{s}{D}}$, dimensionless parameter (dimensionless)

$B i=\frac{k_{l} R}{D_{l}}=\frac{k_{f} R}{D_{e}}$, Biot number (dimensionless)

$a_{l}=\frac{4 \pi R^{2} N_{\mathrm{part}}}{V_{\text {bulk }}}$, specific area of the particles $\left(\mathrm{m}^{-1}\right)$.

Equation (20) is the exact solution of the problem in the Laplace domain.

\section{APPLICATION OF THE MODEL}

\subsection{Data representation and fitting in the Laplace domain}

The time evolution of the bulk concentration $c_{\text {bulk }}(t)$ is experimentally measured. It can be represented by some ad hoc functions for which the Laplace transform is known. As an example, a four-parameter double exponential has been selected [eq. (21)]. Many other time descriptions would be possible and we used some other descriptions, but the best $\chi^{2}$ was obtained when using the double exponential description and this prompted our choice.

$$
c_{\text {bulk }}(t)=a+b \mathrm{e}^{-f t}+\left[c_{\text {bulk }}(t=0)-a-b\right] \mathrm{e}^{-d t} .
$$

The Laplace transform of eq. (21) is

$$
\bar{c}_{\text {bulk }}=\frac{a}{s}+\frac{b}{f+s}+\frac{\left[c_{\text {bulk }}(t=0)-a-b\right]}{d+s} .
$$

domain of $s$ selected is $10^{-5}<s<10^{0.5}$, a choice that will be discussed later.

Thus we fit the numerical values of $s c_{\text {bulk }}$, the Laplace transform of the experimental values computed according to eq. (22) to the exact solution in the Laplace domain. We find that using 40 values of $s$ per decade is in general appropriate. We finally obtain the best values for the kinetic parameters $k_{l}, D_{l}$ and $D$.

\section{MATERIALS AND METHODS}

\subsection{Human serum albumin}

Human serum albumin (Sigma Chemie, Switzerland, product number A-1653) was used without further purification. Its characteristics (van der Scheer, 1978) are reported in Table 1.

\subsection{Buffers}

The experiments were all performed at $T=25^{\circ} \mathrm{C}$ and $\mathrm{pH}=7.0$ with $0.1 \mathrm{M} \mathrm{KCl}$. The buffer used was 0.05 M Tris purchased from Sigma Chemie (Switzerland, product number T-1503 for Trizma ${ }^{\circledR}$ base and T-3253 for Trizma ${ }^{\circledR}$ hydrochloride).

\subsection{Adsorbent}

Blue Sepharose CL-6B (Pharmacia LKB, Uppsala, Sweden) is a group specific adsorbent and Cibacron Blue F3G-A is covalently attached to its cross-linked agarose gel Sepharose CL-6B matrix. The mean diameter of the particles has been measured by laser diffraction (Particle Sizer M3.0, Malvern Instruments, Malvern, England) and is $93 \mu \mathrm{m}$, confirming the value measured by Boyer (1992a). 
Table 1. Characteristics of HSA (van der Scheer, 1978)

\begin{tabular}{ll}
\hline Concentration in human plasma $\left(\mathrm{kg} / \mathrm{m}^{3}\right)$ & $35-40$ \\
Calculated molecular mass $(\mathrm{kg} / \mathrm{kmol})$ & 66,248 \\
Diffusion coefficient in pure water $\left(25^{\circ} \mathrm{C}\right)\left(\mathrm{m}^{2} / \mathrm{s}\right)$ & $6.9 \times 10^{-11}$ calculated (Young and Carroad, 1980) \\
Isoelectric point $(-)$ & $4.5-5.0$ \\
Extinction coefficient at $280 \mathrm{~nm}\left(\mathrm{~m}^{2} / \mathrm{kg}\right)$ & 0.53 \\
\hline
\end{tabular}

Table 2. Physical properties of Blue Sepharose CL-6B (*molecule used to determine porosity)

\begin{tabular}{lcccccc}
\hline $\begin{array}{l}\text { Particle } \\
\text { mean } \\
\begin{array}{l}\text { diameter } \\
(\mu \mathrm{m})\end{array}\end{array}$ & $\begin{array}{c}\text { Internal } \\
\text { bead } \\
\text { porosity }(-) \\
*(\beta \text {-D-glucose })\end{array}$ & $\begin{array}{c}\text { Internal } \\
\text { bead } \\
\text { porosity }(-) \\
*(\text { human } \\
\text { albumin) }\end{array}$ & $\begin{array}{c}\text { External } \\
\text { bed } \\
\text { porosity }(-) \\
\text { (dextran } \\
\text { T2000) }\end{array}$ & $\begin{array}{c}\text { Archimedes } \\
\text { number }(-) \\
\left(20^{\circ} \mathrm{C}\right)\end{array}$ & $\begin{array}{c}\rho_{\text {app }} \\
\left(\mathbf{k g} / \mathrm{m}^{3}\right)\end{array}$ & $\begin{array}{c}\rho_{p} \\
\left(\mathbf{k g} / \mathrm{m}^{3}\right)\end{array}$ \\
\hline $93 \pm 1$ & 0.95 & 0.55 & 0.38 & $2.0 \pm 0.1$ & 1257 & 72 \\
\hline
\end{tabular}

The porosity of the particles was determined by the measurement of the residence time distribution of two void volume markers in a fixed bed. Dextran T2000 (Pharmacia LKB, Uppsala, Sweden) was supposed to be completely excluded from Blue Sepharose CL-6B and was used to measure the external void volume of the bed. $\beta-\mathrm{D}(+)$ Glucose (Pharmacia LKB, Uppsala, Sweden) was used for the measurement of the total void volume. The internal porosity [eq. (1)] obtained by this technique is 0.95 and the external porosity of the bed is 0.38 . Due to the size exclusion effect of Blue Sepharose CL-6B, the actual internal bead porosity for high molecular weight tracers is less than 0.95 . Boyer and Hsu (1992b) measured the internal bead porosity of Sepharose CL-6B with different proteins showing that $\varepsilon=0.55$ for albumin used as a tracer. We will use this value for porosity in our calculations.

The Archimedes number [eq. (24)] is an important characteristic of particulate solids frequently encountered in the fields of fluidization and sedimentation.

$$
A r=\frac{\rho\left(\rho_{\mathrm{app}}-\rho\right) g d_{p}^{3}}{\mu^{2}}
$$

where $\rho$ is the density of liquid phase, $\rho_{\text {app }}$ is the apparent density of the swollen particles, $g$ is the acceleration of gravity $\left(=9.81 \mathrm{~m} / \mathrm{s}^{2}\right), d_{p}$ is the particle mean diameter and $\mu$ is the viscosity of liquid phase $\left(=1.01 \times 10^{-3} \mathrm{~Pa}\right.$ s at $20^{\circ} \mathrm{C}$ ). $\mathrm{Ar}$ is calculated according to the Stokes correlation [eq. (25)]:

$$
A r=18 \operatorname{Re}_{\mathrm{lim}} \quad(A r<3.6) .
$$

$R e_{\text {lim }}$ is the dimensionless Reynolds number at the terminal velocity:

$$
R e_{1 \mathrm{im}}=\frac{\rho u_{\mathrm{lim}}}{\mu}
$$

where $u_{\text {lim }}$ is the terminal velocity of a single settling particle. $u_{\text {lim }}$ was measured in a glass cylinder containing pure water at $20^{\circ} \mathrm{C}$, which led to $A r=2 \pm 0.1$. $\rho_{\text {app }}$ was calculated from eq. (24). $\rho_{p}$ is the mass of dry resin per unit volume of swollen adsorbent.

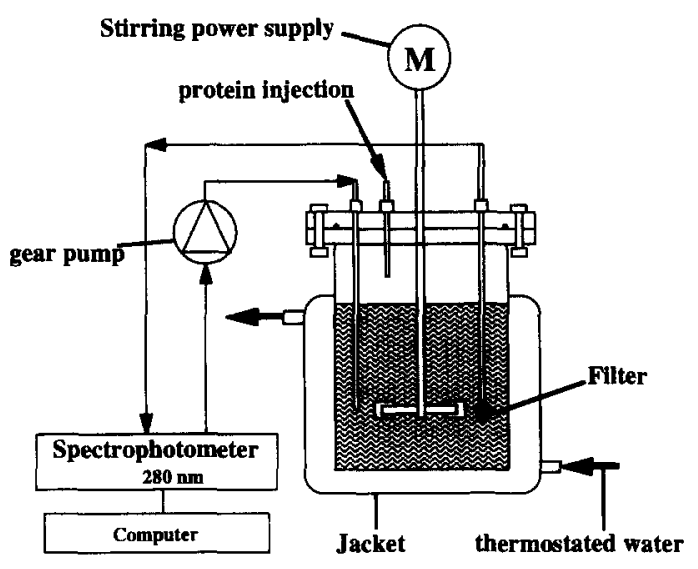

Fig. 1. Apparatus for batch stirred tank experiments.

The summary of the physical properties of Blue Sepharose CL-6B is presented in Table 2.

\subsection{Adsorption kinetics study}

Adsorption kinetics experiments are carried out in a closed stirred tank depicted in Fig. 1.

The vessel has a volume of $3.6 \times 10^{-4} \mathrm{~m}^{3}(6.6 \times$ $10^{-2} \mathrm{~m}$ inner diameter) and is mechanically agitated by an impeller $\left(3.3 \times 10^{-2} \mathrm{~m}\right.$ diameter $)$. The system is jacketed and maintained at $25^{\circ} \mathrm{C}$ by a thermostated water flow. The bulk concentration of HSA was continuously monitored by recycling the liquid phase. The liquid is pumped out of the reaction vessel through a specially designed filter to a quartz flow cell so that the suspended adsorbent does not leave the system. The absorbance is monitored by a UV visible spectrophotometer (Diode array spectrophotometer 8452A, Hewlett-Packard) every $5 \mathrm{~s}$ at $280 \mathrm{~nm}$ and recorded by a computer before return to the experimental vessel. In order to achieve a fast enough time response, the volume of the recycle was kept as small as possible (approximately $4.5 \times$ $10^{-6} \mathrm{~m}^{3}$ ) and the solution was pumped at a flow rate 
of $0.60 \times 10^{-6} \mathrm{~m}^{3} / \mathrm{s}$. At the beginning of the experiments, $0.9 \mathrm{~g}$ of dry Blue Sepharose CL-6B ( $N_{\text {part }}$ $\cong 3 \times 10^{7}$ ) was placed in the tank and equilibrated with $1.50 \times 10^{-4} \mathrm{~m}^{3}$ Tris buffer under soft stirring (50 rotations per minute) during $30 \mathrm{~min}$. Agitation is then stopped and the adsorbent beads settle at the bottom of the vessel. Absorbance acquisition is switched on and $1 \mathrm{ml}$ of a $20 \mathrm{~kg} / \mathrm{m}^{3}$ HSA solution $(0.05 \mathrm{M}$ Tris $\mathrm{pH}=7,0.1 \mathrm{M} \mathrm{KCl}$ ) is gently added in the liquid. After approximately $10 \mathrm{~s}$ the stirring is abruptly switched on $(330 \mathrm{rpm})$. Bulk-phase concentration reaches its maximum (which can be predicted by mass balance) and the adsorption process begins. Data acquisition is stopped when equilibrium is attained. The adsorbent particles showed no sign of damage due to agitation, even after periods of $10 \mathrm{~h}$.

\subsection{Adsorption equilibrium study}

In a typical adsorption kinetics experiment, equilibrium is reached after $5000 \mathrm{~s}$ and the corresponding value of absorbance is recorded. Mass balance on HSA gives the value of the adsorbed amount of protein (expressed as the weight of adsorbed HSA per unit weight of dry adsorbent). Subsequently, $1 \mathrm{ml}$ of $20 \mathrm{~kg} / \mathrm{m}^{3}$ HSA solution is again added to the system. When equilibrium is reached again, the value of absorbance is noted and $q$, the adsorbed quantity of HSA, is calculated. The same procedure is repeated until adsorbent is saturated. The resulting adsorption isotherms were found to fit well to the Langmuir-type expression as given in eq. (27).

\section{RESULTS AND DISCUSSION}

\subsection{Adsorption isotherms}

The adsorption isotherm is determined to estimate the concentration range for which a linear adsorption isotherm can be assumed and to estimate the adsorption equilibrium constants by independent measurements. The result can be used to serve as a control of the fitted kinetic parameters by comparison or to reduce the fitting kinetic parameters to two $\left(D_{e}\right.$ and $\left.k_{l}\right)$.

A typical result for adsorption isotherm measurement is shown in Fig. 2. The set of equilibrium data was fitted to the Langmuir model [eq. (27)] by nonlinear least-squares regression.

$$
q(\mathrm{eq})=\frac{q_{m} c_{\text {bulk }}(\mathrm{eq})}{K_{d}+c_{\text {bulk }}(\mathrm{eq})} .
$$

The results for the equilibrium parameters are given in Table 3. In the kinetic experiments, the upper value for $c_{\text {bulk }}(t)$ was $0.14 \mathrm{~kg} / \mathrm{m}^{3}$ and therefore (see Fig. 2) the isotherm was considered to be linear in the experimental domain.

We know from the preceding theoretical considerations that $D_{l} / D=1+\left(\rho_{p} K / \varepsilon\right)$. Since $\rho_{p}=72 \mathrm{~kg} / \mathrm{m}^{3}$, we obtain (from Tables 2 and 3) $D_{l} / D=79.6$.

\subsection{Adsorption kinetics: application of the model and numerical computation}

5.2.1. Application of the model. A kinetic adsorption experiment provides a set of values for $c_{\text {bulk }}(t)$ as shown in Fig. 3 (for graph legibility reasons, only 100 points are shown). In an experiment lasting $5000 \mathrm{~s}$, 1000 values equidistant in time are measured. The data are fitted by a double exponential [eq. (21)]. Figure 3 represents the data, the fit and the residual to the fit.

The parameters obtained in the fit are used to represent the data in the Laplace domain via eq. (22) multiplied by $s$ (see Fig. 4). In general, we compute 40 values of $s c_{\text {bulk }}$ per decade in a selected domain of $s$. For graph legibility only 50 points are shown. The parameters $D, D_{e}$ and $k_{f}$ are obtained by least-squares fitting.

The domain of $s$ where the fitting is performed must be appropriately chosen. From a theoretical point of view, the calculation of the Laplace transform of $c_{\text {bulk }}(t)$ requires the evaluation of an integral from $s=0$ to $s \rightarrow \infty$.

The formal correspondence between $s$ and $t$ does not associate a specific value of $t$ to a specific value of $s$. In spite of this, it is patent that the experimental time domain does not cover the entire domain of $s$. We only know from theorems (23) that $s=0$

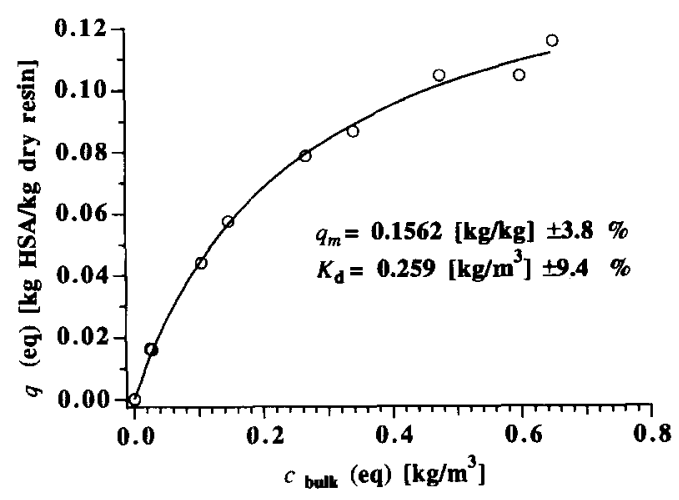

Fig. 2. Adsorption isotherm for HSA on Blue Sepharose CL-6B (pH $=7.0, \quad 0.05 \mathrm{M}$ Tris- $\mathrm{HCl}$ buffer, $0.1 \mathrm{M} \mathrm{KCl}$, $T=25^{\circ} \mathrm{C}$.

Table 3. Equilibrium parameters $\left(\mathrm{pH}=7,0.1 \mathrm{M} \mathrm{KCl}, T=25^{\circ} \mathrm{C}\right.$ )

\begin{tabular}{lccr}
\hline \multicolumn{1}{c}{ Parameter } & Symbol & Value & Standard error (\%) \\
\hline Maximum binding capacity & $q_{m}$ & $0.156(\mathrm{~kg} / \mathrm{kg})$ & 3.8 \\
Apparent dissociation constant & $K_{d}$ & $0.260\left(\mathrm{~kg} / \mathrm{m}^{3}\right)$ & 9.4 \\
Linear adsorption isotherm constant & $K=q_{m} / K_{d}$ & $0.600\left(\mathrm{~m}^{3} / \mathrm{kg}\right)$ & 13.2 \\
\hline
\end{tabular}




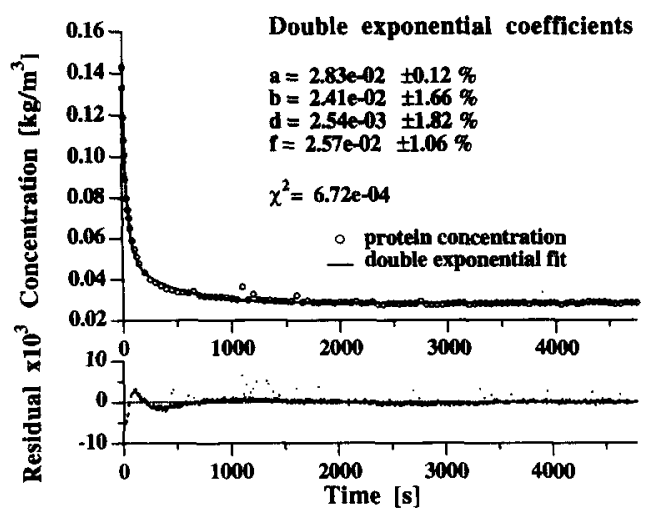

Fig 3. Fitting of experimental data ( 957 points) by double exponential [eq. (21)]. Only 100 points are shown.

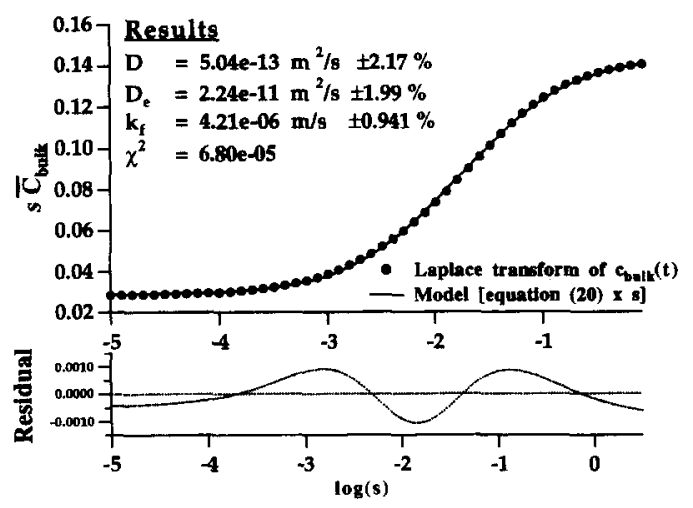

Fig. 4. Fitting of the Laplace transform of the experimental data ( 200 points) by model equation $(20) \times s$. (Only 50 points are shown.)

corresponds to the high time limit $t \rightarrow \infty$, and that inversely $s \rightarrow \infty$ corresponds to the low time limit $t \rightarrow 0$.

The upper value of $s$ can be selected considering the definition of the Laplace transform of $c_{\text {bulk }}(t)$ which can be estimated by discretization with respect to time:

$$
\begin{gathered}
\bar{c}_{\text {bulk }}=\int_{0}^{\infty} \mathrm{e}^{-s t} c_{\text {bulk }}(t) \mathrm{d} t \Rightarrow \\
\bar{c}_{\text {bulk }} \approx \sum_{i=0}^{\infty} \mathrm{e}^{-s t_{1}} c_{\text {bulk }}\left(t_{i}\right) \Delta t .
\end{gathered}
$$

If $\Delta t$ is taken as the time between two measurements $(5 \mathrm{~s})$ the preceding equation becomes

$$
\begin{aligned}
\bar{c}_{\mathrm{bulk}} & \approx \sum_{i=0}^{\infty} \mathrm{e}^{-s t_{i}} c_{\text {bulk }}\left(t_{i}\right) \Delta t=c_{\text {bulk }}(t=0) \Delta t \\
+ & e^{-s 5} c_{\text {bulk }}(5) \Delta t+\cdots+\mathrm{e}^{-s t_{\infty}} c_{\text {bulk }}\left(t_{\infty}\right) \Delta t
\end{aligned}
$$

For example we can state that the second term of eq. (29) cannot exceed $10^{-6}$ times the first term, so that all the following terms of the integral are negli- gible. Introducing the experimental values of $c_{\text {bulk }}(t)$ for the first two terms:

$$
\begin{aligned}
& \mathrm{e}^{-s 5} c_{\text {bulk }}(t=5) \Delta t \leqslant 10^{-6} \cdot c_{\text {bulk }}(t=0) \Delta t \\
& {\left[c_{\text {bulk }}(t=0)=0.143 \text { and } c_{\text {bulk }}(t=5)=0.133\right]}
\end{aligned}
$$

This determines a possible upper value for $s: s \leqslant 2.8$ and $\log (s) \leqslant 0.45$. Using a greater value of $s$ does not significantly change the estimate of the integral according to eq. (28). In general, we choose to perform the fitting for values of $\log (s) \leqslant 0.5$.

The choice of the lower $s$ value is made under a more simple assumption. We select the lower $s$ value such that $s c_{\text {bulk }}$ approaches the minimum measured $c_{\text {bulk }}(t)$ within $1 \%$. This condition is met at $\log (s)=-4.7$. The value finally chosen is $\log (s)=-5.0$.

A lower bound for $\log (s)$ less than -5 does not affect the parameters obtained. We examined the parameters found for a lower $\log (s)$ limit going from $\log (s)=-5.0$ to -10 , by small steps. The values of the kinetic parameters obtained from the fit did not vary significantly ( $3.5 \%$ for $D_{e}$ and $0.6 \%$ for $k_{f}$ ).

The quality of the extrapolation of the fitting function for long time is of some importance because the Laplace transform calculation takes into account values of $c_{\text {bulk }}(t)$ that have not been measured. Erroneous results could be easily obtained if, at the longest time the measurement is performed, equilibrium is far from being reached.

The value obtained for the film mass transfer coefficient $k_{f}=k_{l} \varepsilon$ is in good agreement with the values usually found for proteins in stirred tank experiments (see Table 4).

The pore diffusion coefficient $D_{l}$ is only about 1.7 times less than the bulk value. In a recent article, Boyer and Hsu (1992b) have developed a correlation to estimate the effective diffusion coefficients $\left(D_{e}\right)$ of various proteins in a cross-linked agarose matrix identical to the one we used in our experiments (Sepharose CL-6B). The effective diffusion coefficient $D_{e}$ is simply related to the pore diffusion coefficient $\left(D_{1}\right)$ by eq. (31):

$$
D_{e}=D_{l} \cdot \varepsilon \text {. }
$$

Using $\varepsilon=0.55$ we compare in Table 5 the results obtained by our technique to the value provided by Boyer's correlation and other literature values.

The value we have found for $D_{e}$ is in good agreement with that predicted by Boyer's correlation $(32 \%$ error) which is expected to be accurate within $25 \%$. The value reported by Arnold (1985) seems somewhat low and may contain some error as stated in the paper of Boyer and Hsu (1992b).

The modified diffusion coefficient $D$ is related to $D_{l}$ via eq. (7). However these two parameters are independently fitted [eq. (20)]. Using the parameters obtained from the fit, we find for the ratio $D_{l} / D=81.1$. This value is almost identical to that obtained via eq. (7) $\left(D_{t} / D=79.6\right)$. 
Table 4. Values of $k_{f}$ for various adsorption systems

\begin{tabular}{|c|c|c|c|}
\hline Reference & Protein $(\mathrm{kg} / \mathrm{kmol})$ & Adsorbent & $k_{f}(\mathrm{~m} / \mathrm{s})$ \\
\hline Horstmann and Chase (1989) & $\begin{array}{c}\mathrm{IgG} \\
150,000\end{array}$ & Sepharose & $4.0 \times 10^{-6}$ \\
\hline Firouztale and Scott (1992) & $\begin{array}{c}\text { Insulin } \\
5600\end{array}$ & $\begin{array}{c}\text { Amberchrome } \\
\text { CG-71MS }\end{array}$ & $5.0 \times 10^{-6}$ \\
\hline Skidmore et al. (1990) & $\begin{array}{c}\text { BSA } \\
66,300\end{array}$ & Sepharose FF & $5.0 \times 10^{-6}$ \\
\hline This work & $\begin{array}{c}\text { HSA } \\
66,248\end{array}$ & $\begin{array}{l}\text { Blue Sepharose } \\
\text { CL-6B }\end{array}$ & $4.2 \times 10^{-6}$ \\
\hline
\end{tabular}

Table 5. Values of $D_{\mathrm{e}}$ found in the literature compared to the results of this study

\begin{tabular}{|c|c|c|c|c|c|}
\hline Reference & $\begin{array}{c}D_{e} \times 10^{11} \\
\left(\mathrm{~m}^{2} / \mathrm{s}\right)\end{array}$ & Protein & Matrix & Method & \\
\hline Arnold et al. (1985) & 0.96 & Bovine albumin & Sepharose CL-4B & Moment analysis & (Experimental) \\
\hline Boyer and Hsu (1992b) & 1.5 & Bovine albumin & Sepharose CL-6B & - & (Correlated) \\
\hline This work & 2.2 & Human albumin & Sepharose CL-6B & Laplace domain fit & (Experimental) \\
\hline
\end{tabular}

5.2.2. Numerical simulation. In order to evaluate the solution provided by the present Laplace transform method, the numerical resolution of the system for the adsorption in a closed stirred tank has been carried out. The system is described by a set of two differential equations with appropriate boundary conditions.

The first accounts for film mass transfer:

$$
\frac{\mathrm{d} c_{\text {bulk }}}{\mathrm{d} t}=-\frac{k_{l} 4 \pi R^{2} N_{\text {part }}}{V_{\text {bulk }}}\left[\varepsilon c_{\text {bulk }}-c_{i}(R, t)\right] .
$$

The second accounts for pore diffusion inside the particles and has been given previously [eq. (11)]. The boundary conditions are:

At the center of the particle:

$$
\left.\frac{\partial c_{i}}{\partial r}\right|_{r=0}=0
$$

At the surface of the particle:

$$
\begin{aligned}
\left.\frac{\partial c_{i}}{\partial r}\right|_{r=R} & =\frac{k_{l}}{D_{l}}\left[\varepsilon c_{\text {bulk }}-c_{i}(R, t)\right]=\frac{k_{f}}{D_{e}}\left[\varepsilon c_{\text {bulk }}-c_{i}(R, t)\right] \\
& =\frac{k_{f}}{D_{e}}\left[\varepsilon c_{\text {bulk }}-c_{i}(R, t)\right]
\end{aligned}
$$

Furthermore, $c_{i}(r, t=0)=0$.

We used the kinetic parameters for pore diffusion and film mass transfer that we found by the Laplace transform technique. The equations are treated according to the method of lines fully described in the paper of Horstmann (1989). The governing partial differential equations for film and pore diffusion are solved using the program SimuSolv (The Dow Chemical Company, Midland, Michigan, U.S.A.) running on a Sun Sparc station 10. A program defining the input parameters and setting up the discretized differential equations is written in a special ACSL language (Advanced Continuous Simulation Language). Among

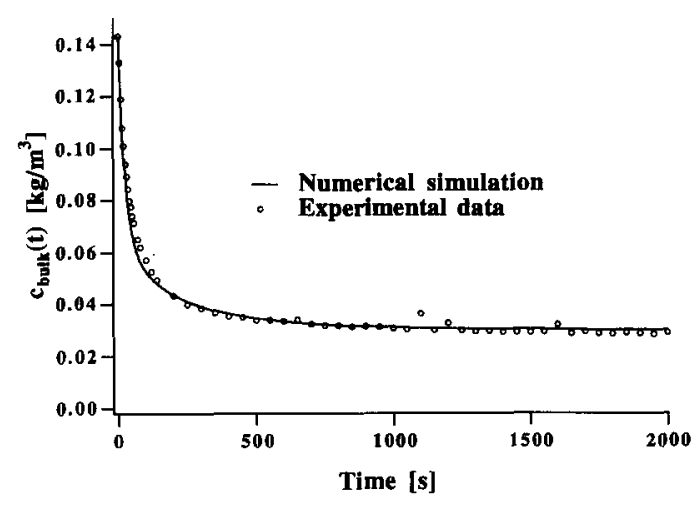

Fig. 5. Numerical simulation with kinetic parameters found by the Laplace transform technique (see Fig. 4) compared to experimental data.

the many integration methods available, a fourthorder fixed-step Runge-Kutta algorithm has been chosen. Point concentrations were transformed into a five-component time-dependent vector, due to the discretization of the space variable. The result of the numerical computation is compared to the experimental data (Fig. 5). It confirms that the kinetic parameters found by the Laplace transform technique provide a good agreement with the experimentally measured time dependence.

\section{CONCLUSIONS}

Kinetic parameters for the adsorption of a protein on porous beads can be obtained via a Laplace transform method. The adsorption of HSA on Blue Sepharose CL-6B has been chosen as a model system. The method uses the Laplace transform technique to solve the partial differential equations system that describes the phenomenon. In the range where a linear adsorption isotherm is valid, by fitting the Laplace 
transform of the exact solution to the Laplace transform of the data, the kinetic parameters obtained by least-squares fitting are in good agreement with the values found in the literature for film mass transfer coefficients and diffusion coefficient in porous adsorbent beads. In order to assert the validity of the values found by this method, a numerical simulation with the obtained model parameters was compared with the experimental results and showed a good agreement. The main advantage of the Laplace transform technique is that the time-consuming numerical approaches are not needed. The parameters are rapidly and easily found via two algebraic fits, one in the time domain followed by another in the Laplace domain. The quality of the results is largely determined by the quality of the fit in the time domain. It may be possible to further improve the results by selecting other satisfactory ad hoc functions to fit the experimental data in the time domain.

Future work will focus on the application of this method to other adsorbents and on the use of other functions that may improve the result in other situations where the four-parameter double exponential does not provide a satisfactory description of the data.

Acknowledgements--This research is supported by the Commission d'Encouragement à la Recherche Scientifique (CERS) and JPS-Chimie (St-Aubin, Switzerland).

\section{NOMENCLATURE}

$a$ double exponential coefficient, $\mathrm{kg} / \mathrm{m}^{3}$ specific surface of the particles, $\mathrm{m}^{-1}$

$a_{1}$

$A$ dimensionless parameter, dimensionless

Ar Archimedes number $\left[=\rho\left(\rho_{\mathrm{app}}-\rho\right) g d_{p}^{3} /\right.$ $\mu^{2}$ ], dimensionless

$b \quad$ double exponential coefficient, $\mathrm{kg} / \mathrm{m}^{3}$

$B i \quad$ Biot number $\left(=k_{l} R / D_{l}=k_{f} R / D_{e}\right)$, dimensionless

$c_{\text {bulk }}(t)$ protein concentration in the bulk of the solution, $\mathrm{kg} / \mathrm{m}^{3}$

$c_{\text {bulk }}(\mathrm{eq})$ protein concentration in the bulk of the solution at equilibrium, $\mathrm{kg} / \mathrm{m}^{3}$

$c_{i}(r, t) \quad$ protein amount per unit volume of swollen particle, $\mathrm{kg} / \mathrm{m}^{3}$

$c_{l i}(r, t)$ protein amount per unit volume of liquid inside a particle, $\mathrm{kg} / \mathrm{m}^{3}$

$d \quad$ double exponential coefficient, $\mathrm{s}^{-1}$

$d_{p} \quad$ mean diameter of the particles, $\mathrm{m}$

$D$ modified diffusion coefficient $\left\{=D_{l} /[1+\right.$ $\left.\left.\left(\rho_{p} K / \varepsilon\right)\right]\right\}, \mathrm{m}^{2} / \mathrm{s}$

$D_{e} \quad$ effective diffusion coefficient $\left(=D_{l} \cdot \varepsilon\right), \mathrm{m}^{2} / \mathrm{s}$

$D_{l}$ diffusion coefficient in the pores of the adsorbent, $\mathrm{m}^{2} / \mathrm{s}$

$f$ double exponential coefficient, $\mathrm{s}^{-1}$

$g$ acceleration due to gravity, $\mathrm{m}^{2} / \mathrm{s}$

$k_{l} \quad$ modified mass transfer coefficient, $\mathrm{m} / \mathrm{s}$

$k_{f}$ mass transfer coefficient, $\mathrm{m} / \mathrm{s}$

$K$ proportionality constant for linear adsorption isotherm, $\mathrm{m}^{3} / \mathrm{kg}$

$K_{d} \quad$ Langmuir equilibrium constant, $\mathrm{kg} / \mathrm{m}^{3}$ $n \quad$ total amount of protein in the system, $\mathrm{kg}$ $n_{\text {bulk }}(t)$ protein amount in the bulk of the solution, $\mathrm{kg}$

$n_{i}(t) \quad$ protein amount inside all the particles, $\mathrm{kg}$

$n_{\text {part }} \quad$ protein amount inside a single particle, $\mathrm{kg}$

$N_{\text {part }}(t)$ number of particles in the system, dimensionless

$q \quad$ global adsorbent loading, $\mathrm{kg} / \mathrm{kg}$

$q(\mathrm{eq})$ global adsorbent loading at equilibrium, $\mathrm{kg} / \mathrm{kg}$

$q_{m} \quad$ maximum binding capacity, $\mathrm{kg} / \mathrm{kg}$

$q_{i}(r, t)$ adsorbed amount of protein per unit weight of dry adsorbent, $\mathrm{kg} / \mathrm{kg}$

$r \quad$ radial coordinate, $\mathrm{m}$

$R$ mean geometrical radius of the particles, $m$

$R e_{\text {lim }} \quad$ Reynolds number at terminal velocity ( $=\rho u_{\text {lim }} d_{p} / \mu$ ), dimensionless

$s \quad$ Laplace variable, $\mathrm{s}^{-1}$

$S_{p} \quad$ surface of a particle, $\mathrm{m}^{2}$

$t$ time, $s$

$t_{\infty} \quad$ long time limit, $s$

$u_{\mathrm{im}} \quad$ terminal velocity of a single settling particle, $\mathrm{m} / \mathrm{s}$

$V_{\text {bulk }} \quad$ volume of the bulk, $\mathrm{m}^{3}$

$V_{\text {void }}$ liquid volume inside a particle, $\mathrm{m}^{3}$

$V_{\text {part }} \quad$ volume of a single particle, $\mathrm{m}^{3}$

$V_{p} \quad$ volume of a solvent swollen particle, $\mathrm{m}^{3}$

\section{Greek letters}

$\Delta t \quad$ time between two measurements, $\mathrm{s}$

$\varepsilon \quad$ porosity, dimensionless

$\mu \quad$ viscosity, $\mathrm{Pa} s$

$\rho_{\text {app }} \quad$ apparent particle density, $\mathrm{kg} / \mathrm{m}^{3}$

$\rho_{p} \quad$ mass of dry resin per unit volume of adsorbent, $\mathrm{kg} / \mathrm{m}^{3}$

$\rho$ density of liquid, $\mathrm{kg} / \mathrm{m}^{3}$

$\Phi(t)$ amount of protein entering one particle per unit time, $\mathrm{kg} / \mathrm{s}$

\section{Superscript}

$\bar{x} \quad$ variable $x$ in the Laplace domain

\section{REFERENCES}

Arnold, F. H., Blanch, H. W. and Wilke, C. R., 1985, Analysis of affinity separations: the characterization of affinity columns by pulse techniques. Chem. Engng J. Biochem. Eng. J. 30, B25-B36.

Arve, B. H. and Liapis, A. I., 1987, Modeling and analysis of biospecific adsorption in a finite bath. A.I.Ch.E. J. Am. Inst. Chem. Engng 33, 179-193.

Boyer, P. M. and Hsu, J. T., 1992a, Effects of ligand concentration on protein adsorption in dye ligand adsorbents. Chem. Engng Sci. 47, 241-251.

Boyer, P. M. and Hsu, J. T., 1992b, Experimental studies in restricted protein diffusion in agarose matrix. A.I.Ch.E. $J$. 38, 259-272.

Casillas, J. L., Martinez, M., Addo-Yobo, F. and Aracil, J., 1993, Modelling of the adsorption of cephalosporin $C$ on modified resins in a stirred tank. Chem. Engng J. 52, B71-B75.

Chase, H. A., 1984, Prediction of the performance of preparative affinity chromatography. J. Chromatogr. 297, 179-202. 
Christoffel, E. G., 1989, Laboratory Studies of Heterogeneous Catalytic Processes, Studies in Surface Science and Catalysis, Vol. 42. Elsevier, Amsterdam.

Firouztale, E., Scott, A. P., Dalvie, S. K. and Von Blohm, G. M., 1992, Experimental and theoretical study of key parameters of adsorption on reverse phase macroporous resins. A.I.Ch.E. Symp. Ser. 88, 25-33.

Horstmann, B. J. and Chase, H. A., 1989, Modelling the affinity adsorption of immunoglobulin $\mathrm{G}$ to protein $\mathrm{A}$ immobilised to agarose matrices. Chem. Engng Res. Des. 67, 243-254.

McKay, G., Blair, H. S. and Gardner, J., 1983, Rate studies for the adsorption of dyestuffs onto chitin. J. Colloid Interface Sci. 95, 108-119.

Rosen, J. B., 1952, Kinetics of a fixed bed system for solid diffusion into spherical particles. J. Chem. Phys. 20, 387-394.
Ruthven, D. M., 1984, Principles on Adsorption and Adsorption Processes. Wiley, New York.

Skidmore, G. L., Horstmann, B. J. and Chase, H. A., 1990, Modelling single-component protein adsorption to the cation exchanger S Sepharose FF. J. Chromatogr. 498, 113-128.

Somers, W., Van't Riet, K., Rozie, H., Rombouts, F. M. and Visser, J., 1989, Isolation and purification of endo-polygalacturonase by affinity chromatography in a fluidized bed reactor. Chem. Engng J. Biochem. Engng J. 40, B7-B19.

van der Scheer, A. T., 1978, Adsorption of plasma proteins: adsorption behaviour on apolar surfaces and effect on colloid stability. Dissertation, University of Twente.

Young, M. E. and Carroad, P. A., 1980, Estimation of diffusion coefficients of proteins. Biotechnol. Bioengng 22, 947-955. 